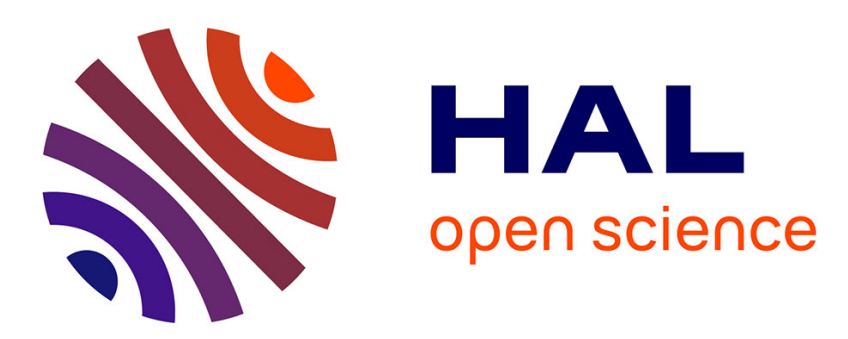

\title{
Optical performance of Bragg gratings fabricated in Ti:LiNbO3 waveguides by focused Ion beam milling
}

Kamal Ghoumid, Richard Ferriere, Badr-Eddine Benkelfat, Brahim Guizal, Tijani Gharbi

\section{- To cite this version:}

Kamal Ghoumid, Richard Ferriere, Badr-Eddine Benkelfat, Brahim Guizal, Tijani Gharbi. Optical performance of Bragg gratings fabricated in Ti:LiNbO3 waveguides by focused Ion beam milling. Journal of Lightwave Technology, 2010, 28 (23), pp.3488-3493. 10.1109/JLT.2010.2080258 . hal00583018

\section{HAL Id: hal-00583018 https://hal.science/hal-00583018}

Submitted on 17 Apr 2021

HAL is a multi-disciplinary open access archive for the deposit and dissemination of scientific research documents, whether they are published or not. The documents may come from teaching and research institutions in France or abroad, or from public or private research centers.
L'archive ouverte pluridisciplinaire HAL, est destinée au dépôt et à la diffusion de documents scientifiques de niveau recherche, publiés ou non, émanant des établissements d'enseignement et de recherche français ou étrangers, des laboratoires publics ou privés. 


\title{
Optical Performance of Bragg Gratings Fabricated in Ti:LiNbO ${ }_{3}$ Waveguides by Focused Ion Beam Milling
}

\author{
Kamal Ghoumid, Richard Ferriere, Badr-Eddine Benkelfat, Senior Member, IEEE, Brahim Guizal, and \\ Tijani Gharbi
}

\begin{abstract}
In this paper we propose an efficient technique for micromachining lithium niobate that is used in Ti-diffused waveguides. The use of Focused Ion Beam (FIB) etching allows obtaining homogeneous periodic microstructures. Bragg gratings with a period of $1.05 \mu \mathrm{m}$ and an aspect ratio of 6:1 (depth-to-half period ratio) have been achieved. A reflectivity greater than $95 \%$ associated with a bandwidth at half maximum of about $100 \mathrm{~nm}$ within a window centered at $1550 \mathrm{~nm}$, is demonstrated for a Bragg grating of period $\Lambda=1.8 \mu \mathrm{m}$ and a length of $144 \mu \mathrm{m}$, in good agreement with theoretical predictions.
\end{abstract}

Index Terms-Bragg grating, focused ion beam, integrated optics, lithium niobate, waveguide.

\section{INTRODUCTION}

$\mathbf{M}$ ANY applications of Bragg reflectors have been developed in the field of biomedical and optical communication systems. Such Bragg gratings (BGs) are highly desirable for providing narrow-spectrum filters, mirrors, couplers, taps and sensing elements that define the basic functions required. BGs have been also employed in wavelength multiplexers and demultiplexers to realize in-line optical filters, especially notch filters and wavelength-specific reflectors given their dispersion compensation properties [1]-[4]. These periodic structures recently have attracted much interest since the discovery of photonic crystals. It is well known that a photonic crystal has some photonic bandgaps in which optical waves with frequencies within them are not allowed to propagate [5]. More recently, it has also been shown that a one-dimensional

K. Ghoumid is with Femto-ST Département d'Optique P.M. Duffieux (LOPMD) UMR CNRS 6174, Université de Franche-Comté, 25030 Besançon Cedex, France, and also with Ecole Nationale des Sciences Appliquées d'Oujda (Maroc), Udjah 6000, Morocco (e-mail: kamal.ghoumid@univ-fcomte.fr; kghoumid@ensa.ump.ma).

R. Ferriere and T. Gharbi are with Femto-ST Département d'Optique P.M. Duffieux (LOPMD) UMR CNRS 6174, Université de Franche-Comté, 25030 Besançon Cedex, France (e-mail: kamal.ghoumid@univ-fcomte.fr; richard.ferriere@univ-fcomte.fr; tijani.gharbi@univ-fcomte.fr).

B.-E. Benkelfat is with Institut Telecom, Telecom Sud-Paris, Samovar UMR, INT-CNRS 5157, 91011 Evry Cedex, France (e-mail: Badr-Eddine. Benkelfat@int-edu.eu).

B. Guizal is with GES UMR CNRS 5650, Université Sciences et Techniques du Languedoc Montpellier 2, 34095 Montpellier Cedex 05, France (e-mail: brahim.guizal@ges.univ-montp2.fr). plane Bragg reflector can be omnidirectional which reflected at all incident angles and for both TE and TM polarizations.

BGs integrated with optical waveguides in lithium niobate $\left(\mathrm{LN}, \mathrm{LiNbO}_{3}\right)$ can be applied in the development of various integrated optic devices. This can be justified by the fact that the $\mathrm{LN}$ is the reference material in the field of integrated optics owing to its large electro-optical, nonlinear and piezoelectric coefficients as well as for exhibiting mechanical and chemical stability [6].

BGs in LN have been developed using different methods such as: photorefractive technique [7], [8], electron-beam lithography and etching [9], electron-beam direct writing [9], [10], Reactive Ion Etching (RIE) [11], laser ablation [12] or proton-exchanged technique [13], [14]. One major advantage of the FIB etching technique is the achievement of good submicron scale optical structures in $\mathrm{LiNbO}_{3}$ without resorting to a mask.

In this work, we used the FIB method to achieve BG etching in the $\mathrm{Ti}: \mathrm{LiNbO}_{3}$ waveguides transverse single-mode. This method has already been employed to etch sub-micrometric one-dimensional structures in $\mathrm{LiNbO}_{3}$ or 2D-photonic crystals [15], [16]. In comparison with more standard methods, FIB milling has the advantage of inducing direct etching without any additional technological steps. Indeed FIB is a high capacity nanostructuring tool providing direct, local and controlled waveguide property modifications. Such conditions are ideal to obtain precise waveguide refractive index corrugations and homogeneous BGs.

The BGs at low index corrugation $d n$ can be treated by the coupled modes theory, one can then calculate the coupling coefficient, reflectivity, and bandwidth by mathematical expressions based on Maxwell's equations. Although this theory has a domain of validity, and it does not apply rigorously to BGs with a high difference index (corrugation), but it still gives an idea about the relationship between the parameters mentioned above. That is to say, the growth of reflectivity or bandwidth is strongly linked to an increased coupling coefficient, which can be obtained either by a strong corrugation $d n$, or by increasing the depth etching $l$ or by increasing the periods number $N$ or Bragg order $m$, or a combination $d n, l, N$ and $m$. The well-behaved control of these parameters undoubtedly led to the achievement of good mirrors and filters based BGs. This point was also discussed in this paper by making a Ti: $\mathrm{LiNbO}_{3}$ waveguide different types of BGs in trying to change the periods number $N$, the depth etching $l$ and the Bragg order $m$. Losses due to the method used for etching of BGs are also discussed in this paper. 


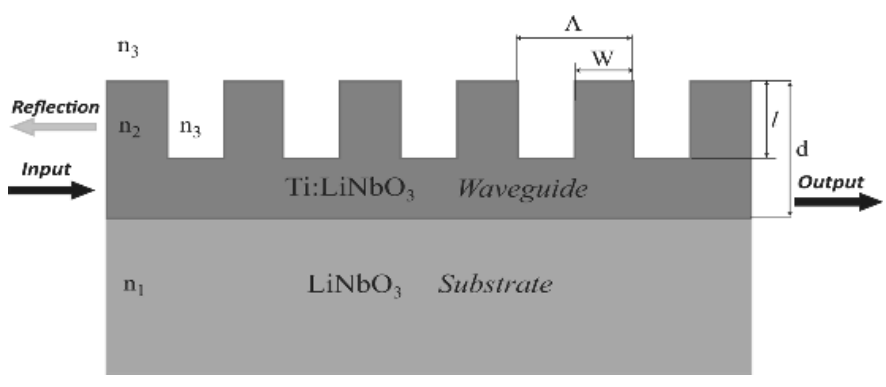

Fig. 1. Basic scheme of a Bragg grating etched in a $\mathrm{Ti}: \mathrm{LiNbO}_{3}$ waveguide $\left(n_{2}>n_{1}, n_{3}=1\right)$.

\section{PRINCIPE AND PERFORMANCES}

\section{A. Principe}

A BG is generally a multi-layered optical structure consisting of two alternating media with differing refractive indexes and fulfilling the so called Bragg condition [17]. Generally it is used to achieve very high reflection of light around a given central wavelength. This concept has been extended to optical waveguides by introducing periodic corrugations.

Fig. 1 shows such a structure with, grating depth $l$ and grating width $W$. With these notations the Bragg condition is written:

$$
2 n_{\mathrm{eff}} \Lambda=m \lambda_{B}
$$

Where $n_{\mathrm{eff}}$ is the effective index of the propagating mode, $m$ the Bragg order, $\Lambda$ Bragg period and $\lambda_{B}$ is the Bragg wavelength. If the condition mentionned in (1) is satisfied, complex amplitudes corresponding to reflected field contributions arising from different parts of the grating are phase matched, so that they can constructively add up [18], [19].

\section{B. Recalls of the Performances}

The performance of a high-quality BG can be described through the following equations:

$$
\begin{aligned}
\Re_{\mathrm{Max}} & =\tanh ^{2}(\kappa L) \\
\Delta \lambda & =\frac{\lambda_{B}^{2}}{n_{\mathrm{eff}} L} \sqrt{1+\left(\frac{\kappa L}{\pi}\right)^{2}}
\end{aligned}
$$

where $\kappa$ is the coupling coefficient between the contrapropagating modes, $L$ the overall grating length with $L=N \Lambda$, where $N$ is the total number of periods. We recall that (2) is a result of the coupled mode theory [20]-[22], but the latter has a certain domain of validity, it is not effective in the case of BGs with high index difference (corrugation). Equations (2) and (3) correspond to the maximum of reflexion $\Re_{\text {Max }}$ and its width at half maximum respectively. In order to obtain maximum reflectivity with a high order Bragg grating, it will be necessary to implement a periodic dielectric waveguide with a significant index and/or length perturbation. The filter bandwidth is inversely proportional to the length of the grating provided the product $\kappa L \ll \pi$. The $n_{\mathrm{eff}}$ value can be controlled via parameters related to the techniques of thermal diffusion during the waveguide fabrication.

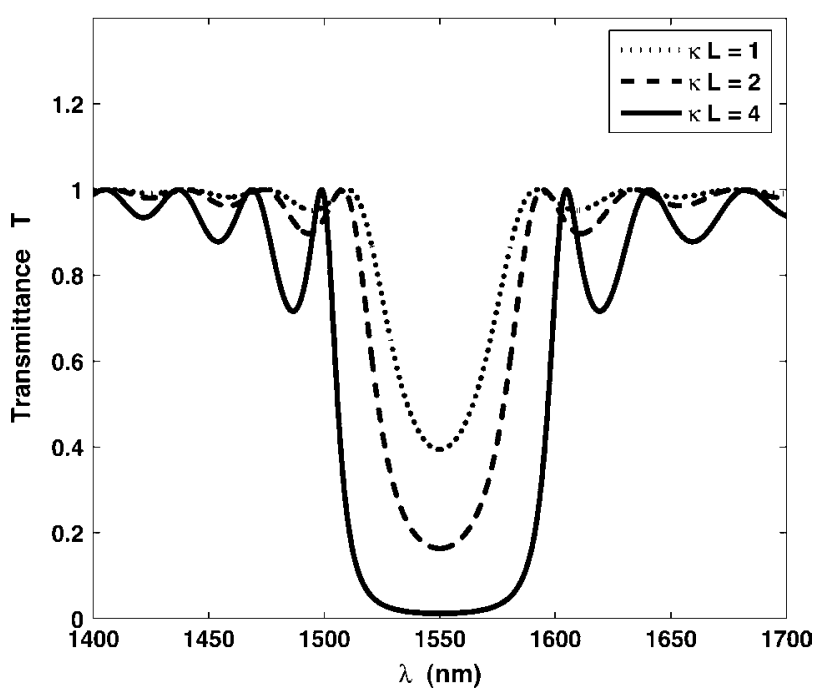

Fig. 2. Transmission versus wavelength for different values of $\kappa L$.

There is a maximum reflectivity $\Re_{\text {Max }}$ corresponds to a minimum transmission $T_{\min }$, which can be written as follows:

$$
T_{\min }=1-\Re_{\mathrm{Max}}-l_{\mathrm{loss}}
$$

where $l_{\text {loss }}$ represents the different types of losses.

As it well known that any increase in the product $\kappa L$ will give a minimum transmission $T_{\mathrm{Max}}$ and high bandwidth $\Delta \lambda$. This is illustrated in Fig. 2 by simulation the Transmittance versus wavelength for different values of $\kappa L$.

It results from simulation that the increase in product $\kappa L$ leads necessarily to obtaining a minimal transmittance (maximum reflectivity), this increase can be obtained by increasing the depth etching $l$ or by increasing the periods number $N$ or Bragg order $m$, or a strong corrugation $d n$, or a combination $d n, l, N$ and $m$. This result is interesting for the perfect control of this component, it is general, and it was shown by the coupled modes theory, the matrix approach [23], [24] (low or high corrugation) and also by the methods based on the Maxwell's equations.

As mentioned in the introduction, good control of these parameters BGs, leads to the achievement of good filters $(<0.4$ $\mathrm{nm})$ and good reflectors $(>50 \mathrm{~nm})$. These filters and reflectors are based an integrated grating in $\mathrm{LiNbO}_{3}$ can be used in many applications since it can be used as a building block in complex optical devices. Among the applications, we quote: Amplitude modulator where means that the dynamic range of the reflected power amplitude can be covered by a narrow translation of the spectrum obtained by electro-optical tuning. BG can also be used in the interrogation systems as a wavelength discriminator or as a tracking filter for a spectral row (i.e., an FBG sensor). Wavelength transducer, Filter trackin. In telecommunications, much different architecture for integrated optical circuits in $\mathrm{LiNbO}_{3}$ can be realized using the Bragg grating as a building block.

It should be noted that the temperature acts on the LN crystal with two different effects: i) thermal expansion who's the expansion coefficient of $\mathrm{LN}$ is $4.110^{-6} / \mathrm{Celsius}$ and $14.110^{-6} / \mathrm{Cel}-$ 
sius as the case where the propagation is parallel or perpendicular to the $\mathrm{C}$ axis. ii) the variation of the refractive index, this is given by the temperature-dependent Sellmeier relations, from which at $1550 \mathrm{~nm}$ one obtains $(d n) /(d T)=8 \cdot 10^{-10} \mathrm{~K}^{-1}$. It is clear that increasing of the temperature induces a shift spectral response. This shift may be interesting if the desired function is filtering. To see the effect of temperature on the phase relationship of a BG, we calculate the derivative of (1) with respect to temperature, we obtain the following equation (for $m=1$ ):

$$
\Delta \lambda_{B}=2\left[\frac{\partial n_{\mathrm{eff}}}{\partial T} \Lambda+n_{\mathrm{eff}} \frac{\partial \Lambda}{\partial T}\right] \Delta T .
$$

For variations between $-40^{\circ} \mathrm{C}$ to $100^{\circ} \mathrm{C}$, we get the wavelength shift of the order $\Delta \lambda_{B} \simeq 1 \mathrm{~nm}$. Note that in our experiments (see below), we obtained reflectors whose bandwidth at half maximum in excess of $60 \mathrm{~nm}$, so such a wavelength shift can be negligible.

The same conclusion remains valid for the wavelength shift due to the difference in index refraction versus temperature, this shift is negligible.

\section{FABRICATION PROCESS}

\section{A. FIB Milling}

Since more than a decade, FIB systems have become common instruments in the field of direct writing at nanometric scale [25]. FIB is a technique used particularly in the semiconductor and materials science fields for site-specific analysis, deposition, and ablation of materials. FIB systems operate in a similar fashion to a scanning electron microscope (SEM) except, rather than a beam of electrons and as the name implies, FIB systems use a finely focused beam of ions (usually gallium) that can be operated at low beam currents for imaging or high beam currents for site specific sputtering or milling.

The milling of samples using FIB is different from the other "traditional" techniques involving lithography and subsequent etching. The essential difference comes from the fact that it uses direct etching without additional processing steps. The only constraint is that the sample surface must be coated and grounded with a metal layer to avoid charge accumulation.

In this paper, we describe a method of direct FIB milling of gratings in Ti-diffused $\mathrm{LiNbO}_{3}$ waveguides through a thin chromium $(\mathrm{Cr})$ layer previously deposited by RF sputtering. The ion beam processing on a solid target induces a significant modification of its structure and physical properties.

However, for a specific amount of $\mathrm{Ga}^{+}$ions, it only produces sputtering of the target material. For the experiments, we use a dual beam Orsay Physics Canion 31/LEO 4400 FIB, the command of the beam deflection being realized by a Raith Elphy Quantum 4.0 driver. The current and ions energy values used for the $\mathrm{LiNbO}_{3}$ sputtering are respectively $300 \mathrm{pA}$ and $30 \mathrm{keV}$.

\section{B. Fabrication of BGs by FIB Milling}

The BG is etched in Ti:LiNbO 3 optical waveguides. The single-mode optical waveguides in a window centered at $1550 \mathrm{~nm}$ were fabricated by standard diffusion of $80 \mathrm{~nm}$ thick
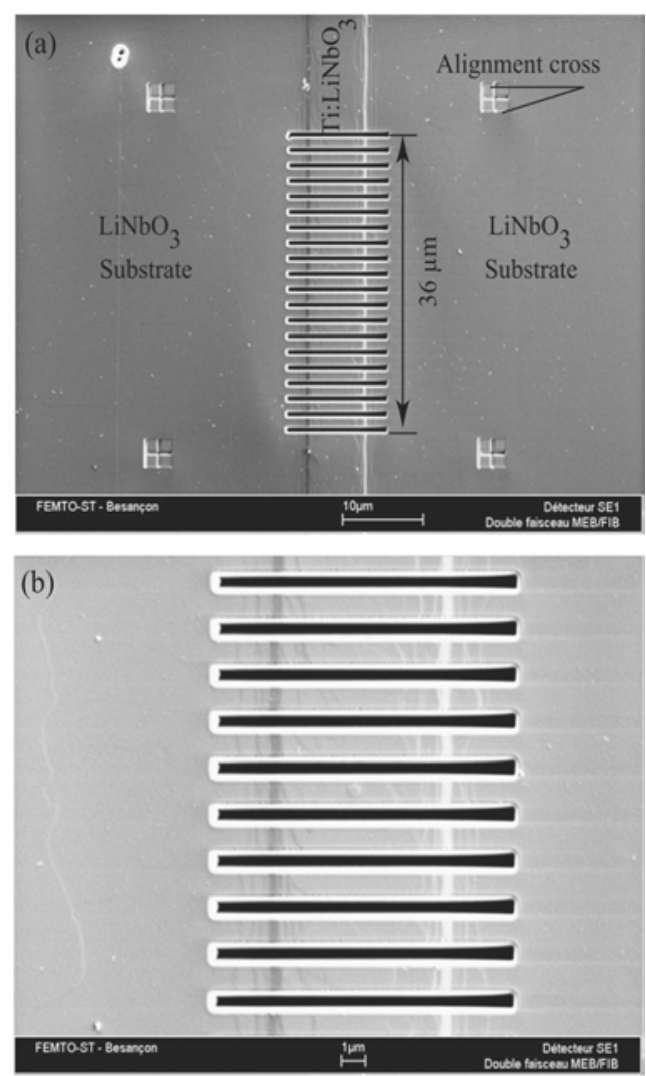

Fig. 3. (a) FIB view of a 20-period Bragg grating ( $\Lambda=1.8 \mu \mathrm{m}, N=20)$ etched in a Ti: $\mathrm{LiNbO}_{3}$ waveguide obtained by standard diffusion: $80 \mathrm{~nm}$ thick and $7 \mu \mathrm{m}$ wide titanium layers at $1020^{\circ} \mathrm{C}$ during $10 \mathrm{~h}$ ). (b) A zoom of $10 \mathrm{Bragg}$ periods.

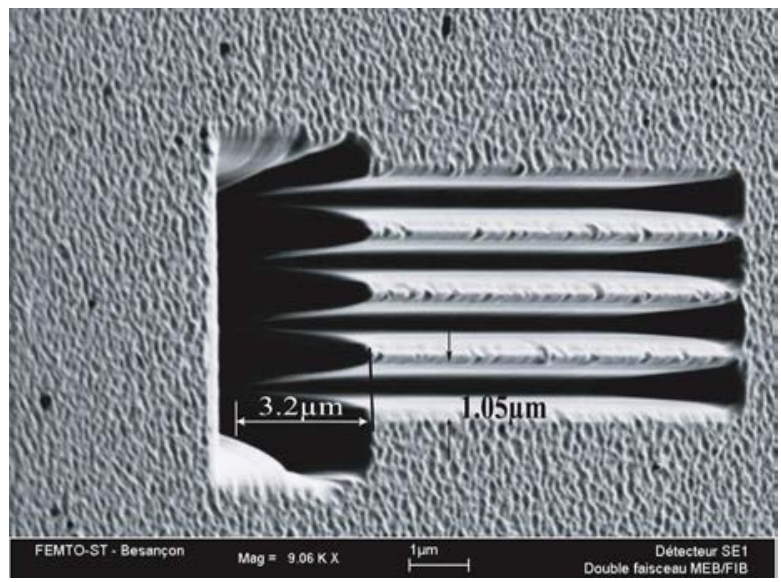

Fig. 4. Visualization of the grating profile obtained by FIB milling with $50^{\circ}$ inclination between substrate plane and ion beam axis. In this case, the grating period is $\Lambda=1.05 \mu \mathrm{m}$ corresponding to an interference order equal to $m=3$. The aspect ratio (depth-to-half period ratio) of the milling can be estimated to be equal at $6: 1$.

and $7 \mu \mathrm{m}$ wide titanium layers at $1020^{\circ} \mathrm{C}$ during $10 \mathrm{~h}$ [14], [26].

Fig. 2 shows a top view of BG with a $7 \mu \mathrm{m}$ width and a period $\Lambda=1.8 \mu \mathrm{m}, N=20$ periods and $l=2 \mu \mathrm{m}$, elaborated by FIB on a X-cut, Z-propagating and TM polarization Ti:LiNbO 3 waveguide. In this situation and using the Sellmeier equation, we have $\left(n_{2}>n_{1}=n_{0}(\lambda=1550 \mathrm{~nm})=2.2112>n_{3}=1\right)$. 


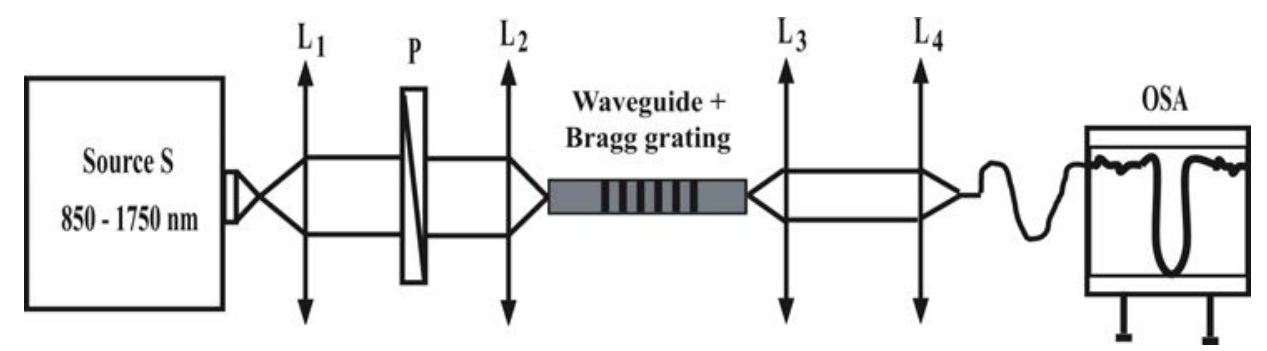

Fig. 5. Measurement setup to characterize Bragg grating transmittance.

We recall that the Bragg period is obtained form (1), $\Lambda=$ $m\left(\lambda_{B}\right) /\left(2 n_{\mathrm{eff}}\right)$. With $\lambda_{B}=1550 \mathrm{~nm}$ and $n_{\mathrm{eff}} \simeq 2.2117$, we get $\Lambda \simeq 1.05 \mu \mathrm{m}$, and $1.8 \mu \mathrm{m}$ for $m=3$ and 5 respectively.

This waveguide is a single transverse mode with lateral dimensions $(4.31 \times 3.62) \mu \mathrm{m}^{2}$ evaluated at $1 / e$ of maximum mode intensities. Clear evidence of homogeneous structures with well reproduced periodicity can be seen in Fig. 3. An FIB-image cross section of the BGs is shown in Fig. 4.

In order to see the shape of the periodic corrugations, the sample has been tilted at $50^{\circ}$ with respect to the FIB axis. The etching profile exhibits a conical shape. This is induced by the material redeposition on the sidewalls during the milling process. Indeed, the etching of $\mathrm{LiNbO}_{3}$ represents however a challenging task, due to the well known resistance of the materiel during the burning process to standard machining methods. The achieved etching depth is approximately 3.2 $\mu \mathrm{m}$ for a period of $1.05 \mu \mathrm{m}$, demonstrating an aspect ratio (depth-to-half period ratio) of 6:1 (see Fig. 4).

\section{RESUlTS AND DisCUSSION}

To assess the spectral response of the previously fabricated BGs, we used the setup depicted in Fig. 5. It consists of a continuous spectrum source $(\mathrm{S})$ in the wavelength range of $\{850-$ $1750\} \mathrm{nm}$ (peak of pump at $1064 \mathrm{~nm}$ ). $L_{1}, L_{2}, L_{3}$ and $L_{4}$ are focusing lenses; $\mathrm{P}$ is a Glan polarizer to selectively probe TM mode, and an optical spectrum analyser (OSA).

We investigate the dependence of optical performances on the following three parameters: the number of periods $N$, the etching depth $l$ and the Bragg order $m$.

First, we would like to emphasize that in our experimental setup there are different types of losses due to [27], [28]: (i) the injection and alignment processes, (ii) material absorption and (iii) BGs small inhomogeneities. In the absence of corrugations the two former types of losses can be estimated from Figs. 6, 7, and 8: the mean difference between curves (a) and (b), to roughly $6 \mathrm{~dB}$. The losses due to etching can be estimated by injecting the signal into a waveguide without corrugation and in another waveguide with a modulation index, this type of loss has been estimated from curves (b) and (c) (on the same figures) at between 2 and $2.5 \mathrm{~dB}$. Thus, knowing the above mentionned losses, in reality, we are going to measure the transmittance at the lens $L_{4}$ output, and so we can also deduce the experimental measured reflectance.

With a BG of period $\Lambda=1.8 \mu \mathrm{m}, m=5$ and $l=2.3 \mu \mathrm{m}$, experimental results of the transmittance for different numbers of periods are given in Fig. 6. It can clearly be seen that as the number of periods $\mathrm{N}$ increases from 30 to 80 , the transmittance

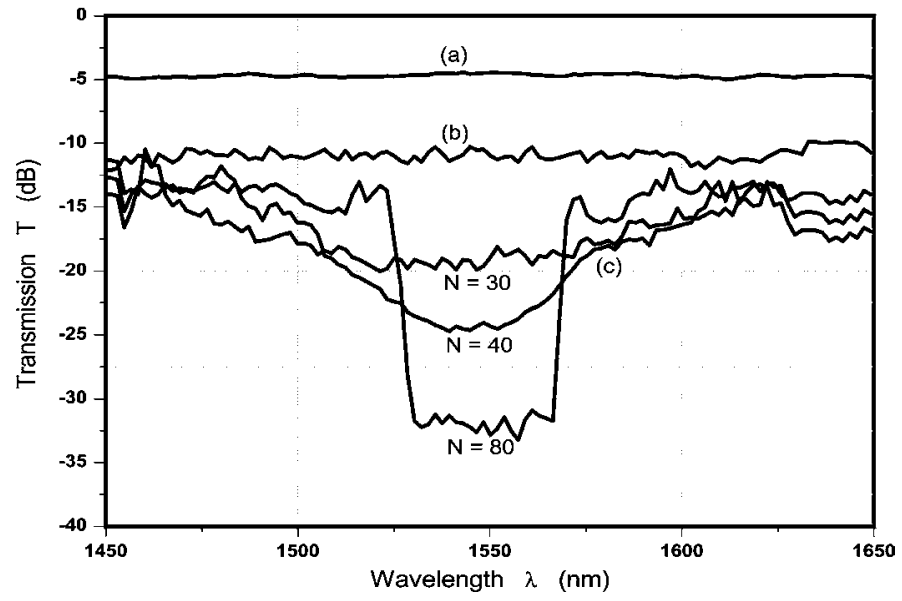

Fig. 6. Transmission versus wavelengths: (a) Source $S$ spectrum, (b) $\mathrm{Ti}^{\mathrm{LiNbO}}{ }_{3}$ waveguide, (c) waveguide with Bragg gratings $(\Lambda=1.8 \mu \mathrm{m}$, $m=5$ and $l=2.3 \mu \mathrm{m}$ ) for different numbers of periods.

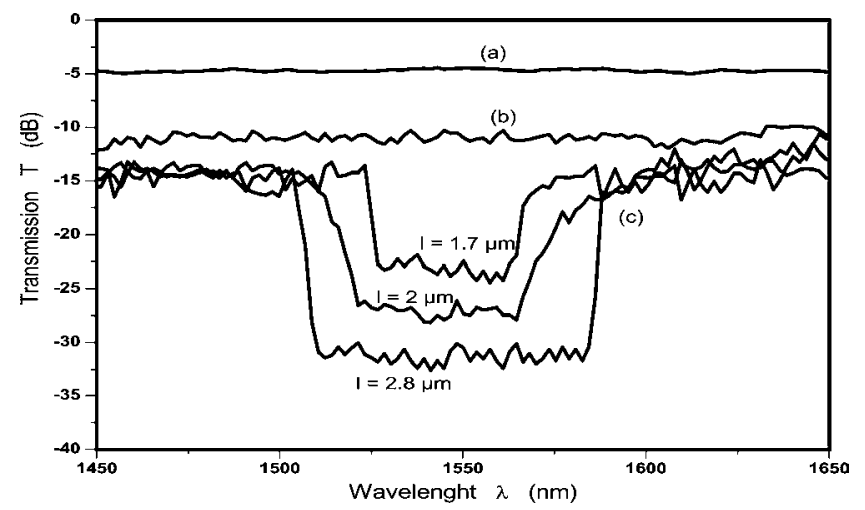

Fig. 7. Transmission versus wavelengths: (a) Source $S$ spectrum, (b) Ti:LiNbO 3 waveguide, (c) waveguide with Bragg gratings $(\Lambda=1.8 \mu \mathrm{m}$, $m=5$ and $N=80$ ) for different milling depths.

decreases from $27 \%(-5.6 \mathrm{~dB})$ to $2 \%(-18 \mathrm{~dB})$. This corresponds to an increase of reflectance from $71 \%$ to $96 \%$ (losses have been taken into account). At the same time, the bandwidth at half maximum $\Delta \lambda$ decreases from 100 to $41 \mathrm{~nm}$.

Fig. 7 shows the measured transmittance for a BGs of period $\Lambda=1.8 \mu \mathrm{m}, m=5, N=80$, for various etching depths. In this case it is seen that the reflectance increases with 1 from $75 \%$ for $l=1.7 \mu \mathrm{m}$ to $92 \%$ for $l=2.8 \mu \mathrm{m}$. However, in this case, the bandwidth at half maximum grows broader with increasing $l$.

Let us now look at the influence of the Bragg order $m$. Various transmittance curves are reported on Fig. 8 for a structure with 


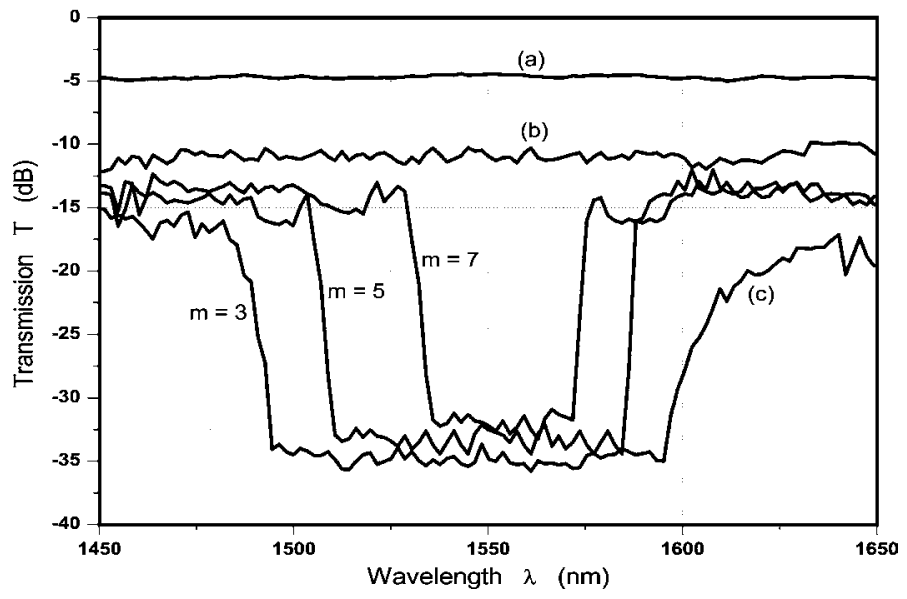

Fig. 8. Transmission versus wavelengths: (a) Source $S$ spectrum, (b) Ti:LiNbO ${ }_{3}$ waveguide, (c) waveguide with Bragg gratings $(l=2.3 \mu \mathrm{m}$ and $N=80$ ) for different values of the Bragg order.

$N=80$ and $l=2.3 \mu \mathrm{m}$. The results show that the reflectance remains nearly constant while the bandwidth at half maximum decreases with increasing $m$ values.

It is important to notify that the FIB etching process introduces an unavoidable roughness in the BGs surface profile. This remaining roughness contributes to the small parasitic peaks that appear in the transmission spectra of Figs. 6, 7, and 8. In addition, the conical etching shape affects the aspect ratio $r=(W) /(\Lambda)$ which increases with the etching depth.

The experimental results nevertheless confirm the theoretical predictions [20]. Furthermore reduced roughness may be achieved through process optimization, e.g., by using lower energy ions.

\section{CONCLUSION}

We have demonstrated that using FIB to etch BGs in a titanium-diffused optical waveguide in LN allows to achieve very high reflectance $(95 \%)$. We have studied the influence of the main parameters of interest: Bragg order $m$, number of periods $N$ and etching depth $l$ on the reflectance.

Nevertheless, this method is effective only for the fabrication of small area structures (e.g., $<100 \mu \mathrm{m}$ ) [29]-[31]. We have also shown that the losses due to BGs are relatively small $(\sim 2$ $\mathrm{dB}$ ) compared to those caused by other techniques like RIE for example.

In addition, FIB milling is performed on a hole-by-hole basis, which implies long process times ( $7-8$ hours for a BG of length $100 \mu \mathrm{m}$, width $7 \mu \mathrm{m}$ and depth $3.2 \mu \mathrm{m}$ ) and eventual drift when fabricating very long BGs. To increase depth, reactive gases can be added to the milling process. For example, by employing $\mathrm{XeF}_{2}$ gas-assisted gallium ion-beam etching in a way the BGs verticality could be optimized [32], indeed $\mathrm{XeF}_{2}$ could help to remove $\mathrm{Nb}$ from the etched substrate decreasing eventually the redeposition. Such experiment is still under development in our laboratory.

\section{ACKNOWLEDGMENT}

The authors would like to thank A. Ramdane for helpful discussions, as well as G. Uliac and R. Salut for technical support which was greatly appreciated.

\section{REFERENCES}

[1] S. Pereira and S. LaRochelle, "Field profiles and spectral properties of chirped Bragg grating Fabry-Perot interferometer," Opt. Exp., vol. 13, no. 6, pp. 1906-1915, 2005.

[2] M. Abtahi, M. Mirshafiei, J. Magné, L. A. Rusch, and S. LaRochelle, "Ultra-wideband waveform generator based on optical pulse-shaping and FBG tuning," IEEE Photon. Technol. Lett., vol. 20, no. 15, pp. 135-137, Sep. 2008.

[3] J. Wang, J. Sun, and Q. Sun, "Proposal for all-optical format conversion based on a periodically poled lithium niobate loop mirror," Opt. Let., vol. 32, no. 11, pp. 1477-1479, 2007.

[4] P. Dekker, M. Ams, G. D. Marshall, D. J. Little, and M. J. Withford, "Annealing dynamics of waveguide Bragg gratings: Evidence of femtosecond laser induced colour centres," Opt. Exp., vol. 18, no. 4, pp. 3274-3283, 2010.

[5] S. Kumar, Awasthi, U. Malaviya, and S. P. Ojha, "Enhancement of omnidirectional total-reflection wavelength range by using one-dimensional ternary photonic bandgap material," J. Opt. Soc. Amer. B, vol. 23, no. 12, pp. 2566-2571, 2006.

[6] R.-H. Kim, J. Zhang, O. Eknoyan, H. F. Taylor, and T. L. Smith, "Narrowband Bragg reflectors in Ti:LiNbO3 optical waveguides," Appl. Opt., vol. 45, no. 20, pp. 4927-4932, 2006.

[7] C. Becker, A. G. T. Oesselke, A. Pape, W. Sohler, and H. Suche, "Integrated optical Ti:Er:LiNbO 3 distibuted Bragg reflector laser with fixed photorefractive grating," Opt. Lett., vol. 23, no. 15, pp. 1194-1196, 1998.

[8] B. Das, R. Ricken, and W. Sohler, "Integrated optical distributed feedback laser with Ti:Er:LiNbO 3 waveguide," Appl. Phys. Let., vol. 82, pp. 1515-1517, 2003.

[9] T. Suhara and H. Nishihara, "Integrated optics components and devices using periodic structures," IEEE J. Quantum Electron., vol. 22, pp. 845-867, 1986.

[10] L. Pierno, M. Dispenza, A. Secchi, A. Fiorello, and V. Foglietti, "A lithium niobate electro-optic tunable Bragg filter fabricated by electron beam lithography," J. Opt. A: Pure Appl. Opt., vol. 10, no. 6, pp. 1-6, 2008.

[11] J. Sochtig, "Ti: $\mathrm{LiNbO}_{3}$ stripe waveguide Bragg reflector gratings," $J$. Electron. Lett., vol. 24, no. 14, pp. 844-845, 1988.

[12] Y. Sidorin and A. Cheng, "Integration of Bragg gratings on $\mathrm{LiNbO}_{3}$ channel waveguides using laser ablation," Electron. Lett., vol. 37, no. 19, pp. 312-314, 2001.

[13] B.-E. Benkelfat, R. Ferriere, B. Wacogne, and P. Mollier, "Technological implementation of Bragg grating reflectors in Ti: $\mathrm{LiNbO}_{3}$ waveguides by proton exchange," IEEE Photon. Technol. Lett., vol. 14, no. 10, pp. 1430-1432, Oct. 2002.

[14] R. Ferriere, B.-E. Benkelfat, J. Dudley, and K. Ghoumid, "Bragg miroir inscription on $\mathrm{LiNbO}_{3}$ waveguides by index microstructuration," $J$. Appl. Opt., vol. 45, no. 15, pp. 1-9, 2006.

[15] F. Lacour, N. Courjal, M.-P. Bernal, A. Sabac, C. Bainier, and M. Spajer, "Nanostructuring lithium niobate substrates by focused ion beam milling," Opt. Mater., vol. 27, no. 8, pp. 1421-1425, 2005.

[16] J. Amet, G. Ulliac, F. I. Baida, and M.-P. Bernal, "Experimental evidence of enhanced electro-optic control on a lithium niobate photonic crystal superprism," Appl. Phys. Lett., vol. 96, p. 103111, 2010.

[17] T. Erdogan, "Fiber grating spectra," J. Lightw. Technol., vol. 15, no. 8, pp. 1277-94, 1997.

[18] K. O. Hill, T. Fujii, D. C. Johnson, and B. S. Kawasaki, "Photosensitivity in optical fiber waveguides: Application to reflection filter fabrication," Appl. Phys. Let., vol. 32, no. 10, pp. 647-649, 1978.

[19] A. Yariv and P. Yeh, Optical Waves in Crystal: Propagation and Control of Laser Radiation. New York: Wiley, 1984.

[20] W. Streifer, D. Scifres, and R. Burnham, "Coupling coefficients for distributed feedback single- and double-heterostructure diode lasers.," IEEE. Quantum Electron., vol. QE-11, no. 11, pp. 867-873, Nov. 1975.

[21] A. Hardy and W. Streifer, "Coupled mode theory of parallel waveguides," J. Lightw. Technol., vol. LT-3, no. 5, pp. 1135-1146, May 1985. 
[22] N. Matuschek, F. X. Kärtner, and U. Keller, "Exact coupled-mode theorys for multilayer interference coatings with arbitrary strong index modulations," IEEE. J. Quantum Electron., vol. 33, no. 3, pp. 295-302, Mar. 1997.

[23] M. Prabhugoud and K. Peters, "Modified transfer Matrix formulation for Bragg grating strain sensors," J. Lightw. Technol., vol. 22, no. 10, pp. 2302-2309, Oct. 2004.

[24] J. Hong, W. Huang, and T. Makino, "On the transfer matrix methode for distributed feedback waveguide devices," J. Lightw. Technol., vol. 10, no. 12, pp. 1860-1868, Dec. 1992.

[25] K. Chaganti, I. Salakhutdinov, I. Avrutsky, G. W. Auner, and J. Mansfield, "Sub-micron grating fabrication on hafnium oxide thin-film waveguides with focused ion beam milling," Opt. Exp., vol. 14, no. 4, pp. 1505-1511, 2006.

[26] R. Schmidth and P. Kaminow, "Metal-diffused optical waveguides in LiNbO 3 ," Appl. Phy., vol. 25, no. 8, pp. 458-460, 1974.

[27] H. Bookey, R. Thomson, N. Psaila, A. Kar, N. Chiodo, R. Osellame, and G. Cerullo, "Femtosecond laser inscription of low insertion loss waveguides in Z-cut lithium niobate," IEEE Photon. Technol. Lett., vol. 19, no. 12, pp. 892-894, Jul. 2007.

[28] F. Rottmann and E. Voges, "Low-insertion-loss, tunable wavelength multiplexer on lithium niobate," Electron. Lett., vol. 23, no. 19, pp. 1007-1008, 1987.

[29] S. Grilli, P. Ferraro, P. De Natale, B. Tiribilli, and M. Vassalli, "Surface nanoscale periodic structures in congruent lithium niobate by domain reversal patterning and differential etching," Appl. Phys. Let., vol. 87, p. 233106, 2005.

[30] P. Ferraro and S. Grilli, "Modulating the thickness of the resist pattern for controlling size and depth of submicron reversed domains in lithium niobate," Appl. Phys. Let., vol. 89, p. 133111, 2006, (2006).

[31] S. Grilli, P. Ferraro, L. Sansone, M. Paturzo, S. DE Nicola, G. Pierattini, and P. DE Natale, "Double-face and submicron two-dimensional domain patterning in congruent lithium niobate," IEEE Photon. Technol. Lett., vol. 18, no. 3, pp. 541-543, Feb. 2006.

[32] S. Yin, "Fabrication of high-aspect-ratio submicron-to-nanometer range microstructures in $\mathrm{LiNbO}_{3}$ for the next generation of integrated optoelectronic devices by focused ion beams (FIB)," Microw. Opt. Technol. Lett., vol. 22, no. 6, pp. 396-398, 1999. 